\title{
Spontaneous Coronary Artery Dissection with Predisposing Fibromuscular Dysplasia: Diagnosed during a Single Cathe- terisation Procedure
}

\author{
AGW de Lepper, MD*, W Aarnoudse, MD, PhD, A Algin, MD, M Winkens, MD and M Magro, MD, PhD \\ Department of Cardiology, Elisabeth-TweeSteden Hospital, Tilburg, Netherlands \\ *Corresponding author: Anouk GW de Lepper, MD, Department of Cardiology, Elisabeth-TweeSteden \\ Hospital, Tilburg, Netherlands
}

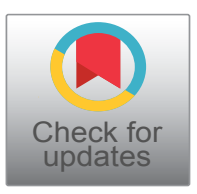

\begin{abstract}
A middle-aged woman presented with a non-ST-segment elevation myocardial infarction which was due to a spontaneous coronary artery dissection of the distal right coronary artery. Angiography of the femoral artery prior to placement of a closure device revealed tortuosity consistent with multifocal fibromuscular dysplasia.
\end{abstract}

\section{History of Presentation}

A 51-year-old female presented in our emergency department with complaints of chest pain with radiation to her jaw and left arm 5 days prior to presentation. Since then, she experienced chest discomfort during exercise with decreasing level of exertion. Physical examination was unremarkable, except for hypertension (RR 153/96 mmHg).

\section{Past Medical History}

Patient had no cardiological medical history and experienced a spontaneous pneumothorax in 2011.

\section{Differential Diagnosis}

Acute coronary syndrome, stable angina and recurrent spontaneous pneumothorax were included in the differential diagnosis.

\section{Investigations}

The electrocardiogram was inconclusive and revealed an incomplete right bundle branch block. Initial cardiac troponin levels were not elevated and other lab results did not reveal any pathological signs. Her chest $\mathrm{X}$-ray showed no signs of a pneumothorax. The exercise test showed frequent, polymorphic, ventricular extrasystole accompanied with chest discomfort. During admission serial cardiac troponin levels revealed a significant rise and fall. Echocardiography demonstrated a normal left ventricular ejection fraction without regional wall motion abnormalities. She was referred for invasive coronary angiography; initially performed through a radial approach. Due to a significant vasospasm the procedure was changed to a femoral approach. The left coronary arteries were not diseased, but revealed tortuosity (Figure 1). The right coronary artery showed distally an occlusion of the ramus descendens posterior (RDP) with TIMI 2 flow. This is consistent with a type 2 spontaneous coronary artery dissection (SCAD) (Figure 2). After the procedure, standard angiographic images were acquired in order to place the intravascular closure device. This revealed a succession of dilations and multifocal stenosis with a string-of-beads appearance consistent with multifocal fibromuscular dysplasia (FMD) (Figure 3).

\section{Management}

The patient was treated medically and immediately started ona beta blocker and dual antiplatelet therapy for 1 year (aspirin and clopidogrel) followed by aspirin monotherapy.

\section{Discussion}

Non-atherosclerotic SCAD is an increasingly impor-

Citation: Lepper AGW, Aarnoudse W, Algin A, Winkens M, Magro M (2021) Spontaneous Coronary Artery Dissection with Predisposing Fibromuscular Dysplasia: Diagnosed during a Single Catheterisation Procedure. Int J Clin Cardiol 8:212. doi.org/10.23937/2378-2951/1410212

Accepted: January 11, 2021: Published: January 13, 2021

Copyright: (C) 2021 Lepper AGW, et al. This is an open-access article distributed under the terms of the Creative Commons Attribution License, which permits unrestricted use, distribution, and reproduction in any medium, provided the original author and source are credited. 


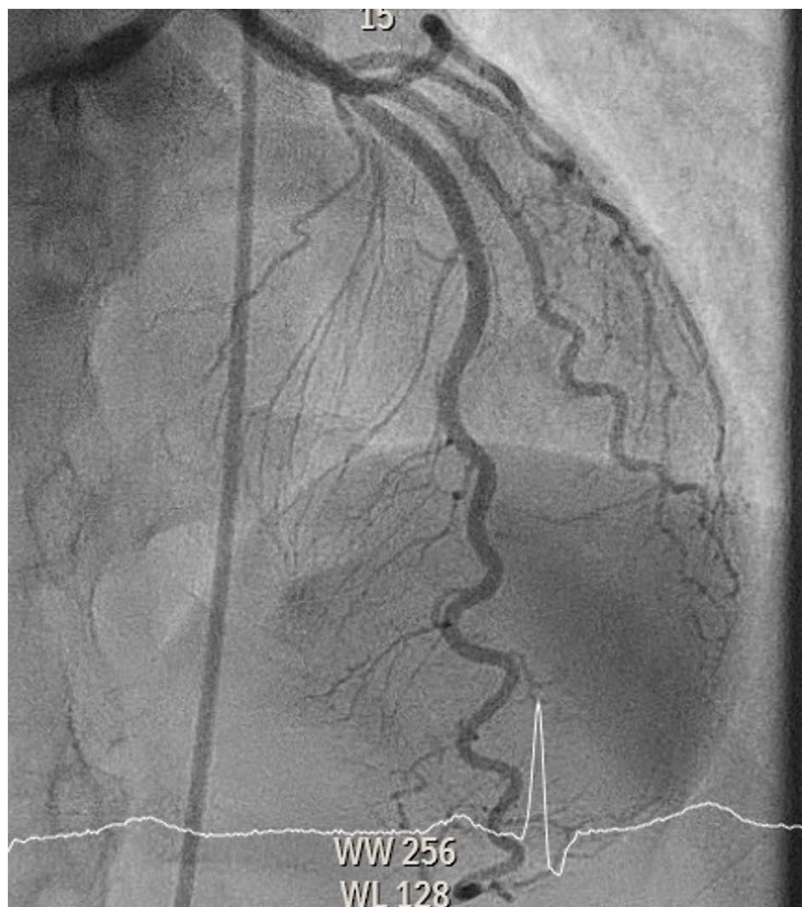

Figure 1: Coronary angiography from left cranial angle, showing tortuosity of left descending artery without significant stenosis.

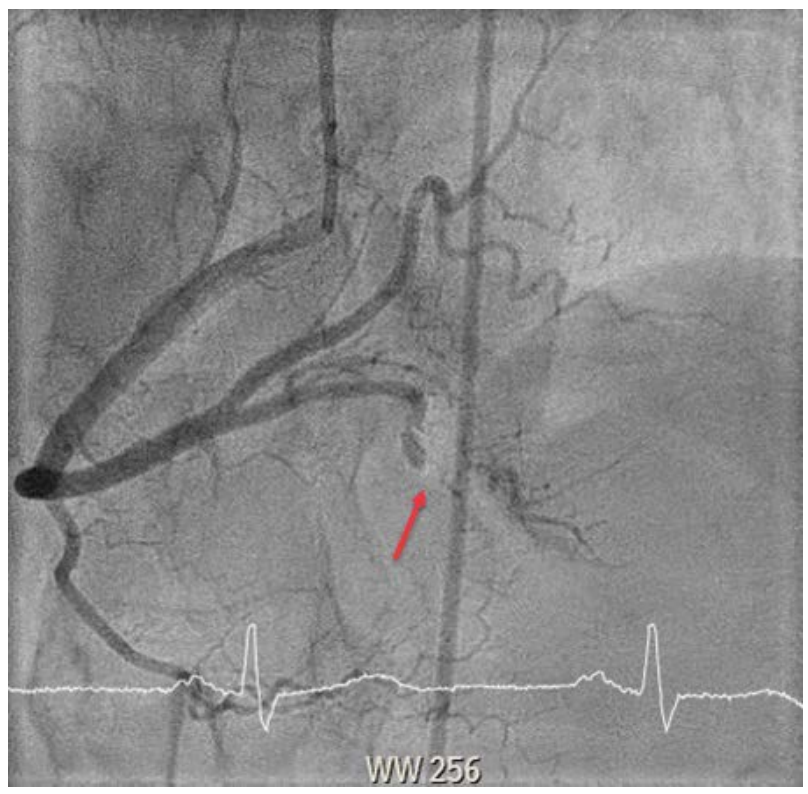

Figure 2: Coronary angiography from right cranial view reveals an occlusion as a result of SCAD distally in the ramus descendens posterior with TIMI 2 flow.

tant cause of myocardial infarction and has for a long time been underdiagnosed and mistreated as atherosclerotic ACS [1]. It is postulated to be the underlying cause of 1.7 to $4 \%$ of acute coronary syndrome while up to $22 \%$ in women under 60 -years-old [2]. The most frequently reported age in woman ranges between 45 and 53 years [1]. The vast majority of SCAD patients are woman (90\%) [3]. Most often (45 to 61\%) the mid to distal segments of the left anterior descending artery is the culprit. During coronary angiography three types of

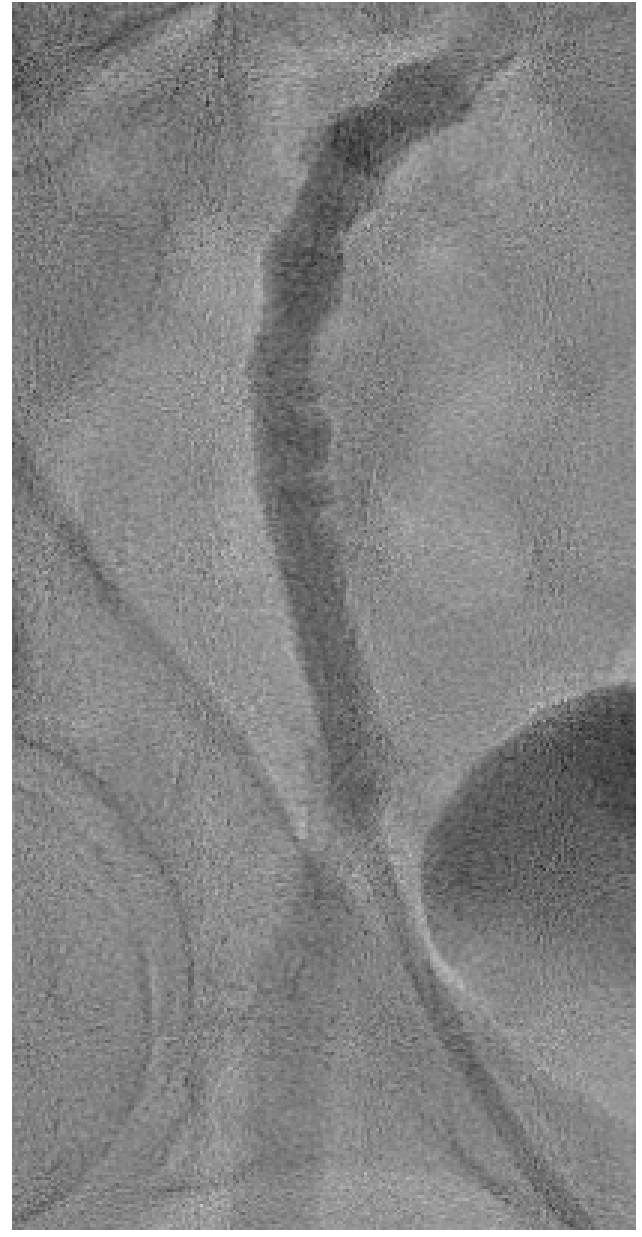

Figure 3: Angiography of femoral artery, showing a stringof-beads appearance with a succession of dilations and multifocal stenosis, consistent with multifocal FMD.

SCAD can be differentiated [4]. Type 1 refers to classical contrast straining with a multiple lumen appearance. Next to this, type 2 SCAD refers to diffuse stenoses with varying length and severity. On the contrary, type 3 mimics atherosclerosis with a focal or tubular stenosis [1]. Precipitating factors are emotional and physical stress in $48 \%$ and $28 \%$ of the cases respectively [5]. The most common predisposing factor is FMD in $62 \%$ of the SCAD patients [5]; as was shown recently by Saw, et al. by using angiography or computed tomography angiography (CTA) of the renal, iliac and cerebrovascular arteries. FMD is a non-atherosclerotic, non-inflammatory vascular condition which can present as a dissection, tortuosity, aneurysm or stenosis in nearly any arterial bed. In total $74 \%$ of patients were screened in the iliac arteries with catheter angiographies. In $55 \%$ of FMD positive patients, there were involvements of iliac arteries. Interestingly, in this study [5], FMD was not a predictor of recurrent SCAD, while hypertension was (HR 2.28). Beta-blockers and aspirin significantly lower the chance of recurrent SCAD (HR 0.39 and 0.36 resp.). Screening for FMD should be done in every patient with palpation and auscultation of abdominal aorta, carotid arteries and peripheral arteries. Imaging from brain to pelvis should be considered with the goal to detect oth- 
er high-risk aneurysms to avoid morbidity and mortality [6]. Therefore, additional catheter-based angiography during coronary angiography of the renal and iliac artery can be done in the same session to diagnose FMD.

\section{Follow-Up}

Cardiac rehabilitation will be performed, and the patient will be followed-up in the outpatient clinic.

\section{Conclusion}

FMD is the most common predisposing factor in SCAD. In cases where femoral access is performed for coronary angiography, additional dedicated iliofemoral artery angiography should be considered in these patients.

\section{Learning Objectives}

Special attention should be given to angiography of iliac arteries during catheterisation in SCAD patients.

\section{References}

1. Hayes SN, Kim ESH, Saw J, Adlam D, Arslanian-Engoren C, et al. (2018) Spontaneous coronary artery dissection: Current state of the science: A scientific statement from the American Heart Association. Circulation 137: e523-e557.
2. Rashid HNZ, Wong DTL, Wijesekera H, Gutman SJ, Shanmugam VB, et al. (2016) Incidence and characterisation of spontaneous coronary artery dissection as a cause of acute coronary syndrome --A single-centre Australian experience. Int J Cardiol 202: 336-338.

3. Adlam D, Alfonso F, Maas A, Vrints C, Committee W (2018) European Society of Cardiology, acute cardiovascular care association, SCAD study group: A position paper on spontaneous coronary artery dissection. Eur Heart J 39: 33533368.

4. Saw J, Aymong E, Sedlak T, Buller CE, Starovoytov A, et al. (2014) Spontaneous coronary artery dissection: Association with predisposing arteriopathies and precipitating stressors and cardiovascular outcomes. Circ Cardiovasc Interv 7: 645-655.

5. Saw J, Humphries K, Aymong E, Sedlak T, Prakash R, et al. (2017) Spontaneous coronary artery dissection: Clinical outcomes and risk of recurrence. J Am Coll Cardiol 70: 1148-1158.

6. Gornik HL, Persu A, Adlam D, Aparicio LS, Azizi M, et al. (2019) First International Consensus on the diagnosis and management of fibromuscular dysplasia. Vasc Med 24: 164-189. 